\title{
Evaluación de la dislexia en la escuela primaria: Prevalencia en español
}

\section{Evaluating dyslexia in primary school children: Prevalence in Spanish}

\author{
María Soledad Carrillo Gallego ${ }^{1}$, Jesús Alegría Iscoa ${ }^{2}$, Pilar Miranda López ${ }^{3}$, \\ Noelia Sánchez Pérez ${ }^{1}$
}

${ }^{1}$ Dpto. de Psicología Evolutiva y de la Educación. Universidad de Murcia (UMU), España. ${ }^{2}$ Lab. Cognition Langage et Développement. Université Libre de Bruxelles (ULB), Bélgica. ${ }^{3}$ EOEP Molina de Segura. Comunidad Autónoma Región de Murcia, España.

Disponible online 31 de agosto de 2011

En este trabajo presentamos un procedimiento secuenciado para la detección de las dificultades lectoras en una muestra representativa de la población escolar constituida por el alumnado de $2^{\circ}, 4^{\circ}$ y $6^{\circ}$ de primaria $(N=2012)$, escolarizado en todos los centros públicos (14) y concertados (5) de un mismo municipio. En la primera fase, un test colectivo de lectura (Test de Eficiencia Lectora TECLE) nos permitió identificar 237 escolares con retraso lector, no explicado por la presencia de otros trastornos (cognitivo, sensorial, conductual, lingüístico, social, etc.). En la segunda fase se aplicaron pruebas de lectura (palabras y pseudopalabras) y de ortografía (palabras con correspondencias fonema-grafema inconsistentes) para seleccionar los casos con retraso lector asociados a dificultades en los mecanismos fonológico y/u ortográfico de base. Los resultados muestran mayor incidencia del retraso lector en $2^{\circ}$ curso de primaria (15.2 \%) frente a la encontrada en $4^{\circ}(9.3 \%)$ y $6^{\circ}(10.9 \%)$. Esta diferencia puede ser explicada por la inclusión en $2^{\circ}$ de alumnos cuyo retraso podría desaparecer sin una intervención especifica. Las dificultades globales de lectura establecidas con el Test de Eficiencia Lectora TECLE se explican en gran parte por dificultades más básicas en descodificación y ortografía.

Palabras clave: Prevalencia de la Dislexia Evolutiva; Dificultades de Aprendizaje de la Lectura; Retraso Lector.

We present a sequential procedure aimed at detecting reading acquisition difficulties. Data were collected from a representative sample $(\mathrm{N}=2012)$ of $2 \mathrm{nd}$, 4th, and 6th graders from all state schools $(\mathrm{n}=14)$ and private schools $(\mathrm{N}=5)$ in a single district. In the first step, a silent reading test (Test de Eficiencia Lectora TECLE) was collectively conducted. It identified 273 children who presented reading difficulties that could not be explained by other causes (cognitive, sensory, behavioural, linguistic, and social). In the second step, the selected sample were administered a reading test (words and pseudo-words), and an orthographic test (words presenting inconsistent phoneme-to-grapheme items) to identify more basic phonological and orthographical deficits which might explain the reading difficulties detected using the Test de Eficiencia Lectora TECLE. The results showed a greater incidence of reading problems in 2nd-grade students $(15.2 \%)$ than in 4 th-grade $(9.3 \%)$ and 6th-grade (10.9\%) students. This difference could be explained by the presence of 2 nd graders with reading difficulties who can overcome their difficulties without any special intervention. We also show that a large part of the global reading delay observed can be explained by difficulties in basic decoding and orthographic abilities.

Keywords: Prevalence Developmental Dyslexia; Reading Acquisition Difficulty; Reading Delay.

Correspondencia: Ma Soledad Carrillo Gallego. Universidad de Murcia, Facultad de Educación. Campus de Espinardo. 30100-Murcia (ESPAÑA). E-mail: mscarri@um.es. Correo electrónico de los otros autores: Jesús Alegría Iscoa: alegria@ulb.ac.be; Pilar Miranda López: mirandalopez.pilar@ gmail.com; Noelia Sánchez Pérez: nsp15716@um.es.

Investigación parcialmente financiada dentro del Proyecto: Evaluación diagnóstica de la dislexia de desarrollo. Programa III - Convenio Universidad de Murcia y Consejería de Educación y Cultura de la Región de Murcia. (Convocatoria 2008). Con el apoyo de la Fundación Séneca -Agencia de Ciencia y Tecnología- de la Consejería de Educación y Cultura de la C.A. de Murcia. Agradecemos la imprescindible colaboración del EOEP de Molina de Segura y la del profesorado y dirección de los CEIP participantes. 
La atención a las necesidades específicas de apoyo educativo (LOE, 2007) incluye al alumnado que presenta dificultades de aprendizaje de la lectura (DAL o dislexia). Paradójicamente, este tipo de dificultad que, según los datos estadísticos de otros países de nuestro entorno más avanzados en su diagnóstico, afecta a una proporción de entre el 5 y el 10\% del alumnado (Flynn y Rahbar, 1994) parece ser el más olvidado en nuestro sistema escolar. Por otra parte, como consecuencia de los informes PISA sobre el rendimiento en lectoescritura y la baja posición que ocupa nuestro país con respecto a otros de nuestro entorno, se ha visto la urgencia de adoptar medidas educativas que mejoren el nivel de competencia en lectura y escritura del alumnado en su conjunto. La adopción de tales medidas pasa necesariamente por un conocimiento diagnóstico de la situación que contemple, entre otras variables y circunstancias, el grado de incidencia en las escuelas de las dificultades de aprendizaje de la lengua escrita. Por otra parte, las medidas educativas que se adopten deben estar fundamentadas en el conocimiento científico y riguroso de los problemas concretos que experimentan los alumnos y que les dificultan el logro de un adecuado nivel de desarrollo lectoescritor. Pero sólo recientemente, con la entrada en vigor de la Ley Orgánica de Educación (LOE), se empieza a contemplar que los alumnos con dificultades de aprendizaje específicas (DA), entre los que se incluyen los que presentan ese tipo de dificultades en el aprendizaje de la lectura (DAL o dislexia), puedan tener una respuesta educativa ajustada a sus necesidades.

Entender en qué consiste la dislexia, saber cómo diagnosticarla y cómo tratarla educativamente es todavía una asignatura pendiente para muchos profesionales de la educación. Esta situación contrasta con el hecho de que la investigación sobre la lectura, realizada desde un planteamiento cognitivo y psicolingüístico, es una de las áreas más y mejor estudiadas de la psicología cognitiva. Las teorías y modelos del procesamiento lector (Perfetti, 1985; Share y Stanovich, 1995; Snowling, 2001), desarrollados en las últimas décadas, aportan en la actualidad una base sólida para la investigación y extensión de nuestro conocimiento sobre el tema. Un ejemplo notable de la incidencia del trabajo de investigación básica de la lectura sobre decisiones clásicamente reservadas a los profesionales de la enseñanza, es el documento redactado por Rayner, Foorman, Perfetti, Pesetsky y Seidenberg (2001) titulado: How psychological science informs the teaching of reading.

Entre los problemas que ocupan el mayor interés de los investigadores se encuentra el referente al desarrollo de la habilidad lectora y las causas de sus trastornos. Particularmente en los casos de dislexia, la atención se ha puesto en los procesos de identificación de palabras de cuyo funcionamiento depende la comprensión de la información transmitida por los textos (Alegría, 2006). La causa fundamental de este problema es que el procedimiento fonológico de lectura no funciona adecuadamente en algunos aprendices que encuentran dificultades para aplicar las correspondencias ortográfico-fo- nológicas de manera exacta y eficiente (automática) y como consecuencia no adquieren representaciones ortográficas plenamente especificadas de las palabras. Esto provoca falta de fluidez en la lectura lo que dificulta la comprensión de frases y textos y se manifiesta también en gran número de errores en la escritura de palabras con grafo-fonemas inconsistentes (Ej. b-v).

Frente a las definiciones anteriores de la dislexia, basadas en criterios de discrepancia y exclusión, las actuales incluyen las características de las dificultades en la lectura y sus causas. Así por ejemplo la Asociación Internacional de Dislexia (IDA) la define como una dificultad específica de aprendizaje de origen neurológico que se caracteriza por dificultades de precisión y fluidez en el reconocimiento de palabras y por problemas de descodificación y deletreo. Estas dificultades tienen su causa en un déficit en el componente fonológico del lenguaje, a menudo inesperado, dado el desarrollo normal de otras habilidades cognitivas y que se ha recibido una enseñanza adecuada (IDA, 2002).

Aunque el núcleo fundamental de la investigación sobre adquisición de la lectura y sus dificultades se ha realizado en lengua inglesa, contamos en la actualidad con importantes investigaciones realizadas en castellano (Calvo, 1999; Carrillo y Alegría, 2009a; Cuetos, Suárez-Coalla y Davies, 2010; Jiménez y Hernández, 2000; Jiménez y Ramírez, 2002; Martínez, 1995; Serrano, 2005; Serrano y Defior, 2005) que identifican los procesos deficitarios en escolares disléxicos. También en esta lengua, y de acuerdo con la Teoría fonológica (Snowling, 2000), los problemas, más o menos graves, tienen su origen en el procesamiento fonológico y repercuten en el ortográfico (Davies, Cuetos y González-Seijas, 2007). Para la evaluación de dichos problemas se requieren pruebas e instrumentos específicos que, de acuerdo con el modelo causal asumido (Hipótesis del déficit fonológico), permitan detectar dónde se sitúan las dificultades de los escolares y su gravedad. La elaboración de protocolos de evaluación y diagnóstico de la dislexia también se ha iniciado recientemente en castellano y en la actualidad disponemos de algunas pruebas adecuadas para este fin en escolares de enseñanza primaria (ver, por ejemplo, Carrillo, 2010; Cuetos, Rodríguez y Ruano, 1996).

Así pues, cualquier avance riguroso en el estudio de las dificultades de aprendizaje de la lectura y el establecimiento de su prevalencia pasa necesariamente por la puesta a punto de instrumentos que puedan evaluar los procesos más relevantes y determinantes del procesamiento lector. Además, una vez identificada la población con DAL, es importante averiguar si se trata o no de una población homogénea, es decir, si el déficit o déficits que presentan son del mismo tipo en todos los casos o si las posibles diferencias lo son sólo en grado o en su tipo o forma. Es necesario, por tanto, que se avance en el campo de la evaluación diagnóstica aplicando técnicas y procedimientos adaptados a la transparencia de la ortografía del castellano y se determine la incidencia y características del retraso lector mo- 
derado y severo en nuestro entorno lingüístico. De esta forma se podrá tomar conciencia de la magnitud del problema y establecer su naturaleza exacta, lo que permitirá establecer un apoyo educativo concebido específicamente para este alumnado.

Con la finalidad de avanzar en el conocimiento de la prevalencia de la dislexia, la presente investigación propone un procedimiento secuenciado de evaluación para su diagnóstico en la población escolar de primaria. Se trata en primer lugar de identificar a los escolares con retraso lector (RL) a través de una prueba de Eficiencia lectora que permite situar al alumno respecto a normolectores de su nivel escolar, y de seleccionar un grupo restringido de niños que incluya la totalidad de los auténticamente disléxicos. A continuación, la aplicación de una batería de pruebas complementarias permitirá determinar la causa próxima de las dificultades de los escolares seleccionados. Concretamente nos hemos centrado en la evaluación del funcionamiento de los dos mecanismos básicos de identificación de palabras escritas (vía indirecta fonológica y vía directa ortográfica) con el fin de confirmar que, cuando se trata de dificultades específicas de aprendizaje, el origen del problema se sitúa en dichos procesos específicos de la lectura, y en última instancia poder aportar remedios también específicos.

\section{Participantes \\ Método}

La muestra original está constituida por los 2012 escolares de $2^{\circ}, 4^{\circ}$ y $6^{\circ}$ de primaria de todos los colegios públicos (14) y concertados (5) del municipio de Molina de Segura (Murcia). En la $1^{\text {a }}$ Fase del estudio (ver procedimiento, más adelante) pudieron ser evaluados 1894 escolares en Eficiencia lectora, 14 no lo fueron por desconocimiento del idioma (alumnado extranjero de reciente incorporación al sistema educativo) o por alguna discapacidad grave, y el resto faltó a clase en las fechas en las que se realizaron las pruebas. En las Tabla 1 se muestra al alumnado evaluado en la $1^{\text {a }}$ fase del estudio, distribuidos por curso y según la categoría en la que fueron clasificados. Los de origen inmigrante con desfase curricular, así como los diagnosticados con Necesidades Educativas Especiales (NEE) y aquellos que por razones de índole socio-cultural también presentaban desfase curricular, aunque fueron evaluados en la primera fase, no se consideraron objeto de nuestro estudio. Sin embargo, sí fueron considerados como escolares con posible dificultad específica en lectoescritura los que presentaban algún tipo de alteración leve en el lenguaje o estaban diagnosticados con trastorno de atención con o sin hiperactividad (TDAH).

Tabla 1. Alumnado participante en la $1^{\text {a }}$ Fase.

\begin{tabular}{|c|c|c|c|c|c|c|c|c|}
\hline Curso & $\begin{array}{c}\text { Sin condiciones } \\
\text { especiales }\end{array}$ & Repetidores & $\begin{array}{c}\text { Dificultades leves } \\
\text { lenguaje }\end{array}$ & TDAH & $\begin{array}{c}\text { Origen } \\
\text { inmigrante }\end{array}$ & NEE & Problemática social & Total \\
\hline $2^{\circ}$ & 469 & 24 & 11 & 3 & 96 & 21 & 13 & 637 \\
\hline $4^{\circ}$ & 471 & 31 & 8 & 3 & 89 & 21 & 8 & 631 \\
\hline $6^{0}$ & 419 & 63 & 3 & 1 & 92 & 27 & 21 & 626 \\
\hline Total & 1359 & 118 & 22 & 7 & 277 & 69 & 42 & 1894 \\
\hline
\end{tabular}

En la $2^{\text {a }}$ fase participaron 233 alumnos de los 237 identificados con retraso lector (LR) en la $1^{\text {a }}$ fase y 166 normolectores
(NL) seleccionados de las mismas aulas de procedencia de los lectores retrasados (ver Tabla 2).

Tabla 2. Alumnado con Retraso Lector (RL), clasificado por condición, y grupo control de Normolectores (NL) participantes en la $2^{\mathrm{a}}$ Fase.

\begin{tabular}{c|cccc|cc|c}
\hline Curso & $\begin{array}{c}\text { LR sin condiciones } \\
\text { especiales }\end{array}$ & LR Repetidores & $\begin{array}{c}\text { LR Dificult. leves } \\
\text { lenguaje }\end{array}$ & LR TDAH & Total LR & NL & Total evaluados \\
\hline $\mathbf{2}^{\mathbf{0}}$ & 78 & 17 & 6 & 3 & $\mathbf{1 0 4}$ & $\mathbf{7 7}$ & $\mathbf{1 8 1}$ \\
$\mathbf{4}^{\mathbf{0}}$ & 46 & 13 & 1 & 1 & $\mathbf{6 1}$ & $\mathbf{4 7}$ & $\mathbf{1 0 8}$ \\
$\mathbf{6}^{\mathbf{0}}$ & 43 & 24 & 1 & & $\mathbf{6 8}$ & $\mathbf{4 2}$ & $\mathbf{1 1 0}$ \\
\hline Total & $\mathbf{1 6 7}$ & $\mathbf{5 4}$ & $\mathbf{8}$ & $\mathbf{4}$ & $\mathbf{2 3 3}$ & $\mathbf{1 6 6}$ & $\mathbf{3 9 9}$ \\
\hline
\end{tabular}

\section{Procedimiento y pruebas utilizadas}

El estudio que presentamos consta de dos fases de evaluación sucesivas, la $1^{\text {a }}$ fase cuyo objetivo es la identificación de los casos con retraso lector no asociado a discapacidad, desconocimiento de la lengua o factores socio-culturales, y la $2^{\mathrm{a}}$ fase dirigida a seleccionar, entre los lectores retrasados (LR), los casos cuyo fracaso es probablemente debido a dificultades específicas en los mecanismos de identificación de palabras. Además, como grupo de comparación fue seleccionada una muestra de normolectores (NL) de las mismas aulas de procedencia de los casos con retraso (ver su distribución por curso en Tabla 2).
En la $1^{\text {a }}$ fase se administró, colectivamente en los grupos completos de aula, la prueba TECLE de Eficiencia lectora (Carrillo y Marín, 1997) que combina comprensión y rapidez y permite establecer el nivel global de competencia lectora de cada alumno. Consta de 64 frases a las que les falta la última palabra y debajo de cada una de ellas se presentan, en posición variable, cuatro opciones de respuesta (palabra adecuada, palabra de ortografía similar pero no adecuada semántica o sintácticamente, pseudopalabra similar gráficamente a la opción correcta y pseudopalabra similar fonológicamente a dicha opción). A medida que la prueba avanza las frases aumentan en 
longitud y complejidad morfosintáctica y las palabras que las forman disminuyen en familiaridad. Las instrucciones, dadas verbalmente por el examinador, incluyen la realización previa de dos frases de ensayo y advierten que se dispone de 5 minutos para completar el mayor número de frases que se pueda. La puntuación es el número de frases completadas correctamente en el tiempo estipulado.

En la $2^{\mathrm{a}}$ fase se administró individualmente a cada escolar de los dos grupos seleccionados (LR y NL) una prueba de Lectura de palabras y pseudopalabras. En ella se considera tanto la exactitud como la velocidad en la lectura en voz alta de tres listas de 12 palabras frecuentes de distinta longitud (2, 3 y 4 sílabas) y de tres listas de 12 pseudopalabras equiparadas en longitud y frecuencia silábica posicional a las de las palabras. Además, se administraron en sesiones diferentes (con una semana de intervalo, al menos) y en pequeños grupos de 8-10 escolares una prueba Dictado de palabras y otra de Decisión ortográfica. En ambas pruebas se incluyen 66 palabras de alta frecuencia, según el diccionario de Martínez y García (2004), que contienen fonemas de escritura inconsistente $(\mathrm{b} / \mathrm{v} ; \mathrm{g} / \mathrm{j}$ seguidas de e-i; h/ no h en posición inicial). En la prueba de Dictado, cada palabra se dicta contenida en una frase en la que ocupa el último lugar; sólo esta palabra debe ser escrita y sólo se puntúa el grafema o grafemas inconsistentes. En la prueba de Decisión ortográfica, cada palabra se presenta escrita junto al pseudohomófono que resulta de sustituir el grafema inconsistente por el alternativo (p.ej. vacaciones - bacaciones) y se debe marcar la forma correcta de la palabra. Por último, se administró el Test de inteligencia de Matrices progresivas de Raven (color).

Los resultados en estas pruebas, que forman parte de la Batería DIS-ESP (Carrillo y Alegría, en preparación) tratan de identificar a los escolares cuyo retraso en el dominio de la lectura es específico de los procesos o mecanismos de identificación de palabras escritas, distinguiéndolos de aquellos otros escolares cuyo problema podría estar justificado por otros motivos tales como baja inteligencia, deficiencias en la comprensión del lenguaje oral o deficiencias en procesos superiores de comprensión del lenguaje escrito.

\section{Resultados}

\section{Resultados de la primera fase}

Para la identificación del alumnado con retraso lector, se calcularon los estadísticos descriptivos básicos (media y desviación típica: $D T$ ) en la prueba de Eficiencia lectora (TECLE) en cada uno de los cursos estudiados $\left(2^{\circ}, 4^{\circ}\right.$ y $6^{\circ}$ de primaria) y se estableció una puntación de corte para cada curso restando a la media correspondiente 1.5 DT. Sólo las puntuaciones del alumnado no afectado de circunstancias especiales fueron consideradas en estos cálculos, los repetidores y los que presentan un desfase curricular importante, ya sea por origen inmigrante, N.E.E. asociadas a discapacidad grave o condiciones socioculturales notablemente desfavorecidas, así como los afectados de TDAH o dificultades leves de lenguaje fueron excluidos de este análisis. La finalidad de estas exclusiones fue obtener un valor promedio y una estimación de la dispersión de los resultados adecuados para la detección de los casos con dificultades específicas en lengua escrita. En la Tabla 3 se muestra la puntuación de corte en el test TECLE para cada curso (7.25, 16.64 y 26.28 en $2^{\circ}, 4^{\circ}$ y $6^{\circ}$ respectivamente) por debajo de la cual un escolar se considera con retraso grave en lectura, si bien, en el caso de escolares repetidores (con mayor edad y años de escolaridad) los límites se establecieron de acuerdo a la edad en cada caso. Nótese que los valores de corte en $4^{\circ}$ y $6^{\circ}$ corresponden aproximadamente a las medias de $2^{\circ}$ y $4^{\circ}$ respectivamente. Esto indica que los cortes adoptados en este estudio coinciden aproximadamente con el criterio clásico que considera que los disléxicos presentan dos años de retraso en lectura.

Tabla 3. Resultados por curso en la prueba de Eficiencia Lectora (TECLE) y puntuaciones de corte.

\begin{tabular}{c|ccccc}
\hline \multicolumn{2}{c}{$\begin{array}{c}\text { Grupo sin condicionantes } \\
\text { especiales }\end{array}$} & \multicolumn{2}{c}{$\begin{array}{c}\text { Eficiencia lectora } \\
\text { Test TECLE }\end{array}$} & $\begin{array}{c}\text { Puntuación de } \\
\text { corte }\end{array}$ \\
\hline Curso & $\boldsymbol{N}$ & $\begin{array}{c}\text { Edad } \\
\text { (meses) }\end{array}$ & $\boldsymbol{M}$ & $\boldsymbol{D T}$ & $\boldsymbol{M}-\mathbf{1 . 5} \boldsymbol{D T}$ \\
\hline $\mathbf{2}^{\mathbf{0}}$ & 469 & 89.7 & 16.9 & 6.5 & 7.2 \\
$\mathbf{4}^{\mathbf{0}}$ & 471 & 113.5 & 28.4 & 7.8 & 16.6 \\
$\mathbf{6}^{\mathbf{0}}$ & 419 & 137.6 & 41 & 9.8 & 26.3 \\
\hline
\end{tabular}

Como resultado de la aplicación de las puntuaciones de corte establecidas quedaron seleccionados 237 escolares con dificultades en la prueba de Eficiencia lectora. Su distribución por curso y condición se muestra en la Tabla 4 , pudiendo observarse mayor incidencia en $2^{\circ}$ curso que en $4^{\circ}$ y $6^{\circ}$ en todas las condiciones. Además, en cada curso se seleccionó un grupo control de normolectores ( 77 de $2^{\circ}, 47$ de $4^{\circ}$ y 42 de $6^{\circ}$ ) cuyas puntuaciones se encontraban entre el valor de la media de su curso y el límite marcado por la media más 1.5 DT.

Al comparar el grado de incidencia del retraso lector en las distintas condiciones del alumnado se observa que casi la mitad de los repetidores presentan problemas graves de lectura, alcanzando en $2^{\circ}$ un altísimo porcentaje $(75 \%)$. La frecuencia del problema en los casos de dificultades leves de lenguaje, aunque menor que en los repetidores, resulta tres veces mayor que

Tabla 4. Escolares identificados con problemas de Eficiencia lectora por condición y curso.

\begin{tabular}{|c|c|c|c|c|c|c|c|c|}
\hline \multirow{2}{*}{$\begin{array}{c}\text { Curso } \\
2^{\circ}\end{array}$} & \multicolumn{2}{|c|}{ Sin condicionantes especiales } & \multicolumn{2}{|c|}{ Repetidores } & \multicolumn{2}{|c|}{ Dificultades leves lenguaje } & \multicolumn{2}{|c|}{ TDAH } \\
\hline & 79 de 469 & $16.8 \%$ & 18 de 24 & $75.0 \%$ & 6 de 11 & $54.5 \%$ & 3 de 3 & $100 \%$ \\
\hline $4^{\circ}$ & 47 de 471 & $10.0 \%$ & 13 de 31 & $41.9 \%$ & 1 de 8 & $12.5 \%$ & 1 de 3 & $33.3 \%$ \\
\hline $6^{0}$ & 43 de 419 & $10.3 \%$ & 24 de 63 & $38.7 \%$ & 1 de 3 & $33.3 \%$ & 0 de 1 & $0 \%$ \\
\hline Total & 169 de 1359 & $12.4 \%$ & 55 de 118 & $46.6 \%$ & 8 de 22 & $36.4 \%$ & 4 de 7 & $57 \%$ \\
\hline
\end{tabular}


dentro del grupo sin condicionantes especiales $(36.4 \%$ frente al $12.4 \%$, respectivamente), observándose también una mayor incidencia en $2^{\circ}$ curso. Así pues, encontramos que los trastornos leves del lenguaje constituyen un factor de riesgo añadido en la aparición de retraso grave de aprendizaje de la lengua escrita. Lo mismo ocurre con el alumnado con TDAH, en el que los problemas de lectura afectan al 57\% de los participantes, si bien podemos observar que en $2^{\circ}$ curso la incidencia es mayor.

El problema de los escolares identificados en esta $1^{\text {a }}$ fase con retraso lector podría tener diferentes causas, entre ellas que el bajo rendimiento en la prueba de eficiencia lectora refleje un problema en comprensión del lenguaje (falta de vocabulario, sintaxis pobre o incluso dificultades para elaborar proposiciones y establecer las conexiones necesarias entre las mismas que permiten la obtención del significado); en estos casos el problema de comprensión lectora sería consecuencia lógica del problema de comprensión lingüística. En otros casos, el bajo rendimiento en la prueba de lectura puede ser debido a lentitud general en la resolución de tareas escolares, lo que tiene consecuencias negativas en pruebas en las que la velocidad es importante. Pero en este estudio nos interesamos por los problemas específicos de aprendizaje de la lectura que tienen como causa dificultades en la adquisición de los mecanismos de identificación de palabras escritas. Por tanto, la $2^{\mathrm{a}}$ fase se centra en identificar tales casos dentro del grupo previamente identificado con retraso lector. Se trata de aquellos escolares con dificultades, bien en el mecanismo fonológico de lectura, bien en el mecanismo de identificación ortográficalexical, o en ambos. Los resultados en las pruebas de lectura de palabras y pseudopalabras y en las pruebas de decisión ortográfica entre homófonos y dictado permiten identificar estos casos.

\section{Resultados de la segunda fase}

De los 237 escolares con deficiente eficiencia lectora, 233 completaron la evaluación de la $2^{\mathrm{a}}$ fase, junto con los 166 normolectores seleccionados como grupo de comparación (véase Tabla 2). En las Tablas 5, 6 y 7 se presentan los resultados obtenidos por curso en las pruebas administradas en esta $2^{\mathrm{a}}$ fase y también los resultados previos en Eficiencia lectora y la edad promedio de cada grupo. En la prueba de lectura de palabras y pseudopalabras, además de las medidas de exactitud ( $\mathrm{n}^{\mathrm{o}}$ de respuestas correctas en los 36 ítem de cada parte) y tiempo total empleado en la lectura (en segundos) se calcularon índices de eficiencia dividiendo el número de aciertos (incluidas las respuestas con errores leves de silabeo, repeticiones o rectificaciones) por el tiempo empleado. En las dos pruebas ortográficas la puntuación se calculó según la fórmula: $\left(\mathrm{n}^{\mathrm{o}}\right.$ de aciertos $-0.5 * \mathrm{n}^{\mathrm{o}}$ de ítem) $/\left(0.5 * \mathrm{n}^{\mathrm{o}}\right.$ de ítem / 100) con el fin de controlar la probabilidad de respuestas al azar (0.5). Esta puntuación es igual a 0 cuando el sujeto responde al azar, e igual a 100 cuando sus respuestas son siempre exactas.

Se aplicaron análisis de varianza en cada curso para establecer las posibles diferencias entre normolectores (NL) y lectores retrasados (LR). Los resultados indican que las diferencias de edad entre los dos grupos sólo son significativas en $6^{\circ}$ curso $(p=.009)$ debido a la presencia de buen número de repetidores entre los LR. Por otra parte, el nivel intelectual evaluado con el test Raven difiere entre NL y LR tanto en $2^{\circ}$ como en $4^{\circ}$ $(p<.001)$ lo que sugiere que en algunos casos del retraso lector puede estar asociado a bajo C.I.

En cuanto a las variables experimentales de procesamiento lector, los resultados de cada curso muestran importantes diferencias entre normolectores y lectores retrasados en todas ellas. Además, se puede observar que el nivel en lectura de palabras

Tabla 5. Resultados en las Pruebas de la $2^{\mathrm{a}}$ Fase $-2^{\mathrm{o}}$ curso.

\begin{tabular}{|c|c|c|c|c|}
\hline & \multicolumn{2}{|c|}{ Lectores retrasados $(\mathrm{N}=104)$} & \multicolumn{2}{|c|}{ Normolectores $(\mathrm{N}=77)$} \\
\hline & $M$ & $D T$ & $M$ & $D T$ \\
\hline Edad (meses) & 90.62 & 5.00 & 90.61 & 3.55 \\
\hline Eficiencia Lectora (TECLE) & 9.17 & 3.54 & 22.56 & 2.84 \\
\hline Decisión Ortográfica & 23.09 & 19.53 & 62.34 & 19.58 \\
\hline Dictado (inconsistentes) & 34.90 & 9.89 & 70.77 & 18.22 \\
\hline Test Raven & 88.82 & 9.89 & 103.63 & 13.76 \\
\hline Lectura palabras. Aciertos & 29.38 & 6.82 & 35.03 & 1.21 \\
\hline Lectura palabras. Tiempo & 70.85 & 27.54 & 35.23 & 8.08 \\
\hline Lectura palabras. Eficiencia & .54 & .22 & 1.06 & .26 \\
\hline Lectura pseudopalabras. Aciertos & 20.21 & 7.15 & 26.55 & 4.62 \\
\hline Lectura pseudopalabras. Tiempo & 109.75 & 32.52 & 80.73 & 19.42 \\
\hline Lectura pseudopalabras. Eficiencia & .27 & .08 & .42 & .09 \\
\hline
\end{tabular}


Tabla 6. Resultados en las Pruebas de la $2^{\mathrm{a}}$ Fase $-4^{\mathrm{o}}$ curso.

\begin{tabular}{|c|c|c|c|c|}
\hline & \multicolumn{2}{|c|}{ Lectores retrasados $(N=61)$} & \multicolumn{2}{|c|}{ Normolectores $(\mathrm{N}=47)$} \\
\hline & $M$ & $D T$ & $M$ & $D T$ \\
\hline Edad (meses) & 114.77 & 5.44 & 114.19 & 3.6 \\
\hline Eficiencia Lectora (TECLE) & 16.88 & 4.23 & 35.55 & 3.66 \\
\hline Decisión Ortográfica & 50.67 & 24.91 & 92.65 & 7.36 \\
\hline Dictado (inconsistentes) & 56.87 & 21.24 & 93.90 & 6.02 \\
\hline Test Raven & 94.51 & 12.93 & 102.62 & 11.58 \\
\hline Lectura palabras. Aciertos & 32.07 & 3.65 & 35.42 & 0.97 \\
\hline Lectura palabras. Tiempo & 48.26 & 18.74 & 29.53 & 6.79 \\
\hline Lectura palabras. Eficiencia & .80 & .26 & 1.27 & .29 \\
\hline Lectura pseudopalabras. Aciertos & 22.46 & 6.38 & 29.3 & 3.21 \\
\hline Lectura pseudopalabras. Tiempo & 97.28 & 38.25 & 58.00 & 12.26 \\
\hline
\end{tabular}

Tabla 7. Resultados en las Pruebas de la $2^{\mathrm{a}}$ Fase $-6^{\circ}$ curso.

\begin{tabular}{|c|c|c|c|c|}
\hline & \multicolumn{2}{|c|}{ Lectores retrasados $(\mathrm{N}=68)$} & \multicolumn{2}{|c|}{ Normolectores $(\mathrm{N}=42)$} \\
\hline & $M$ & $D T$ & $M$ & $D T$ \\
\hline Edad (meses) & 141.03 & 6.75 & 138.02 & 3.41 \\
\hline Eficiencia Lectora (TECLE) & 25.21 & 3.46 & 45.43 & 4.1 \\
\hline Decisión Ortográfica & 75.40 & 13.85 & 96.90 & 3.51 \\
\hline Dictado (inconsistentes) & 79.99 & 14.20 & 97.01 & 4.24 \\
\hline Test Raven & 95.5 & 11.97 & 100.64 & 12.80 \\
\hline Lectura palabras. Aciertos & 34.53 & 1.42 & 35.81 & 0.45 \\
\hline Lectura palabras. Tiempo & 33.79 & 7.77 & 28.00 & 7.17 \\
\hline Lectura palabras. Eficiencia & 1.10 & .25 & 1.37 & .37 \\
\hline Lectura pseudopalabras Aciertos & 23.94 & 4.95 & 30.21 & 2.91 \\
\hline Lectura pseudopalabras Tiempo & 69.4 & 11.28 & 49.62 & 7.16 \\
\hline
\end{tabular}

y pseudopalabras de los LR de $6^{\circ}$ apenas alcanza al de los NL de $2^{\circ}$, pues aunque muestran mayor velocidad, su mayor número de errores no les permite superar en eficiencia a los normolectores de $2^{\circ}$. En conocimiento ortográfico, los disléxicos de $6^{\circ}$ sí llegan a superar el nivel normolector de $2^{\circ}$ pero quedan muy lejos del nivel normolector de $4^{\circ}$. Consecuentemente, los correspondientes análisis de varianza sobre las medidas de exactitud y eficiencia en lectura de palabras y de pseudopalabras indican que las diferencias entre LR y NL en cada curso son altamente significativas $(p<.0001)$. Resultados similares se obtienen en los correspondientes análisis en las dos medidas de conocimiento ortográfico: decisión ortográfica y dictado. Así pues parece justificada la utilización de estas pruebas en el diagnóstico del retraso lector.

El siguiente paso en nuestro trabajo es identificar de forma más precisa los casos individuales con déficit en el mecanismo fonológico, ortográfico o en ambos, para lo cual seguimos un criterio similar al utilizado en la fase 1. En este caso, los estadísticos de referencia (media y DT) fueron calculados únicamente sobre el grupo de normolectores previamente selec- cionado. El criterio para valorar el mecanismo fonológico se basó en el índice de eficiencia en la prueba de lectura de pseudopalabras, que combina exactitud y tiempo empleado. Para valorar el mecanismo ortográfico se utilizó el promedio de las puntuaciones en las pruebas de dictado y decisión ortográfica. Entre los lectores retrasados, se identificaron con déficit en el mecanismo fonológico aquellos casos en los que el índice de eficiencia en pseudopalabras era inferior al valor de la media de los normolectores del mismo curso menos 1.5 DT. Similarmente, los casos con déficit en el mecanismo ortográfico fueron los que puntuaron por debajo de la media menos 1.5 DT (calculadas para normolectores) en la puntuación promedio de las dos tareas ortográficas.

Como resultado de la identificación anterior, 14 LR sólo presentaban puntuaciones por debajo del límite establecido en lectura de pseudopalabras (Tipo1), 30 las presentaban sólo en la puntuación ortográfica (Tipo2) y 180 las presentaban en ambas puntuaciones (Tipo3), mientras que 9 LR no presentaban puntuaciones por debajo de los límites en ninguna de las dos medidas. Este resultado indica que el $96.1 \%$ de los casos con 
problemas en los mecanismos de lectura de palabras ya habían sido identificados a través del test de Eficiencia lectora (TECLE) y que en el $3.9 \%$ restante, con bajas puntuaciones en este test, no parece que sean los problemas en la identificación de palabras la razón de sus dificultades lectoras. La distribución por curso de la frecuencia de los diferentes tipos de LR se muestra en la Tabla 8.

Tabla 8. Clasificación de los lectores retrasados (LR) por tipo de mecanismo de lectura deficitario.

\begin{tabular}{|c|c|c|c|c|}
\hline & $\begin{array}{c}\text { LR. } 2^{\circ} \\
(n=104)\end{array}$ & $\begin{array}{l}\text { LR. } 4^{\circ} \\
(\mathrm{n}=61)\end{array}$ & $\begin{array}{l}\text { LR: } 6^{0} \\
(n=68)\end{array}$ & $\begin{array}{c}\text { Total } \\
(\mathbf{N}=\mathbf{2 3 3})\end{array}$ \\
\hline Sin dificultades graves & $7(6.7 \%)$ & $2(3.3 \%)$ & & $9(3.9 \%)$ \\
\hline $\begin{array}{l}\text { Diferencias sólo } \\
\text { Fonológicas (Tipo1) }\end{array}$ & $8(7.7 \%)$ & $1(1.6 \%)$ & $5(7.4 \%)$ & $14(6 \%)$ \\
\hline $\begin{array}{l}\text { Diferencias sólo } \\
\text { Ortográficas (Tipo2) }\end{array}$ & $19(18.3 \%)$ & $6(9.8 \%)$ & $5(7.4 \%)$ & $30(12.9 \%)$ \\
\hline $\begin{array}{l}\text { Diferencias Fonológicas } \\
\text { y Ortográficas (Tipo3) }\end{array}$ & $70(67.3 \%)$ & $52(85.2 \%)$ & $58(85.2 \%)$ & $180(77.2 \%)$ \\
\hline
\end{tabular}

Cabe señalar que 7 de los LR sin dificultades graves en los mecanismos de lectura son de $2^{\circ}$ curso y los otros 2 de $4^{\circ}$, lo que parece indicar que en lectores retrasados, la Eficiencia lectora evaluada con TECLE es en los primeros cursos menos dependiente del funcionamiento de los mecanismos de identificación de palabras que lo es en los cursos siguientes.

Los resultados indican por tanto que la mayoría de los LR identificados en la $1^{\text {a }}$ fase $(83 \%)$ presentan un déficit en el mecanismo fonológico de lectura (sean del Tipo1 o del Tipo3) y que además dicho déficit se acompaña de un déficit ortográfico en el $93 \%$ de estos casos (los 180 del Tipo3). La distribución por cursos de la frecuencia de los distintos tipos de dificultades indica que el problema fonológico, como causa del retraso o déficit lector, no disminuye con el aumento de la experiencia lectora, al contrario, en $2^{\circ}$ curso el 18.3 $\%$ de los LR parecen presentar sólo un problema de conocimiento ortográfico mientras que en $6^{\circ}$ este porcentaje disminuye al 5\%. Parece posible que buena parte de ese $18.3 \%$ de LR de $2^{\circ}$ superen su retraso tan sólo con más experiencia lectora.

Teniendo en cuenta los resultados en esta $2^{\mathrm{a}}$ fase, de los 233 escolares evaluados y previamente identificados como LR en la $1^{\text {a }}$ fase, 224 pueden ser considerados con dificultades específicas en el aprendizaje de la lectura, lo que supone un $11.8 \%$ de la población escolar participante en el estudio. La distribución por cursos no es homogénea ya que en $2^{\circ}$ se ha identificado una proporción mayor $(15.2 \%)$ que en $4^{\circ}(9.3 \%)$ y $6^{\circ}(10.9 \%)$. La identificación de escolares con DAL en $2^{\circ}$ podría ser más difícil de precisar dado que algunos escolares aún se encuentran en una fase crítica del desarrollo del mecanismo fonológico, que tiene consecuencias directas en la cantidad y calidad de su práctica lectora y consecuentemente en el desarrollo del léxico ortográfico, mostrando así dificultades del tipo 2. Cabe pensar que son estos casos los que hacen aumentar el porcentaje de escolares identificados con retraso lector.

\section{Discusión}

El objetivo principal de este trabajo era establecer la prevalencia de los problemas de lectura en la enseñanza primaria, utilizando un procedimiento secuenciado y un conjunto de pruebas que permitan evaluar diferentes aspectos de la competencia lectora en el medio escolar. Una de ellas, la más general, evalúa la Eficiencia lectora a través de la lectura silenciosa de frases, sin considerar los mecanismos que permiten al lector alcanzar un nivel particular en esta prueba. Seguidamente se utilizaron otros dos tipos de pruebas con el fin de evaluar los mecanismos básicos, fonológico y ortográfico, implicados en la identificación de palabras escritas. La teoría subyacente a este enfoque, considera que para leer, es decir para comprender una frase escrita, o más generalmente un texto, se necesitan conocimientos lingüísticos generales (vocabulario, morfosintaxis y pragmática) y conocimientos específicos que intervienen en la identificación de las palabras. Ambas son condiciones "sine qua non", lo que significa que cuando se fracasa en una de ellas, la comprensión lectora es necesariamente deficiente.

El procedimiento seguido en nuestro estudio comienza con una fase de detección del retraso lector a través de una prueba de Eficiencia lectora (TECLE). Esta prueba nos permitió rápidamente identificar el grupo de niños que presentaban problemas de lectura. Es interesante señalar que la puntuación de corte que establecimos en nuestro estudio para categorizar un alumno con grave retraso lector (media - 1.5 DT) coincide con la media de la población de escolares inferior en dos cursos. De esta forma, los casos identificados cumplen el criterio clásico de presentar un retraso lector de al menos dos cursos. Los resultados de nuestro análisis identifican con retraso lector al $12.5 \%$ del alumnado. Si la distribución del nivel lector establecida con la prueba de Eficiencia Lectora (TECLE) fuera normal este porcentaje tendría que ser de $6.7 \%$. El exceso del porcentaje observado indica que la distribución del nivel lector no es normal sino que presenta una asimetría en el extremo inferior de la distribución donde se sitúan los disléxicos, lectores con dificultades específicas de aprendizaje, junto a otros lectores retrasados por motivos diferentes.

En cuanto a la incidencia o prevalencia de las DAL o dislexia, los porcentajes absolutos obtenidos tras la $2^{\text {a }}$ fase de evaluación (11.8\% de la muestra estudiada) no pueden ser interpretados de manera directa. El criterio de retraso elegido, similar al utilizado en otros estudios y basado en el nivel de los normolectores de referencia (media menos 1.5 DT), es de nuevo arbitrario, si el criterio hubiera sido más, o menos, estricto, los porcentajes de alumnos identificados hubieran sido diferentes. Jiménez, Guzmán, Rodríguez y Artiles (2009) en un estudio similar en la Comunidad Canaria, identificaron un $8.6 \%$ de escolares con dificultades especificas de aprendizaje de la lengua escrita, correspondiendo el 3.2\% a dificultades sólo en lectura y el $5.4 \%$ restante a dificultades en lectura y ortografía. En nuestro estudio se incluyen entre las DAL ambas categorías, 
puesto que en ambas están presentes las dificultades de lectura. La diferencia en la prevalencia encontrada $(8.6 \%$ frente a $11.8 \%$ ) podría explicarse por diferencias en el procedimiento y criterios de identificación. En el estudio canario fueron los profesores los que valoraron qué alumnos presentaban retraso en lectura y en ortografía para realizar la primera selección de lectores retrasados, mientras que en nuestro caso utilizamos un mismo test de Eficiencia lectora que aporta un criterio más objetivo y homogéneo. Los resultados de esta primera selección fueron del $27.9 \%$ de la muestra estudiada en el caso de la Comunidad Canaria y del $12.5 \%$ en nuestro estudio. Por el contrario, cuando se aplicaron test individuales de lectura de palabras y pseudopalabras (PROLEC en el caso canario) los porcentajes fueron del $8.6 \%$ y del $11.8 \%$, respectivamente en el estudio de Canarias y en el nuestro. Una diferencia importante es que nosotros, junto a la lectura de pseudopalabras, utilizamos las puntuaciones en las pruebas de conocimiento ortográfico, en lugar de las puntuaciones en lectura de palabras, con el fin de poder evaluar con mayor independencia los dos mecanismos de lectura (fonológico y ortográfico-lexical). Además, el criterio de corte utilizado también es diferente en los dos estudios, Jiménez et al. (2009) identifican al 25\% inferior según los baremos de PROLEC, mientras que en nuestro caso se toman únicamente las puntuaciones de los normolectores seleccionados, y se establece el límite en 1.5 DT por debajo de la media. Este procedimiento, basado sólo en puntuaciones de normolectores, resulta pertinente en nuestro caso puesto que el objetivo no es identificar el retraso lector sino valorar el posible "mal funcionamiento" de los mecanismos de identificación de palabras, y ello se hace en relación al funcionamiento de tales mecanismos en los normolectores.

Es importante agregar, que los resultados en la propia prueba de eficiencia lectora (TECLE) ya permiten identificar con un altísimo nivel de acierto a los escolares con dificultades específicas, tal como se constata al aplicar las pruebas de la $2^{\mathrm{a}}$ fase que confirmaron al $96.1 \%$ de los casos. No obstante, cabe esperar que en algunos de estos casos las dificultades en los mecanismos de identificación de palabras se acompañen de bajos niveles en el dominio de la lengua oral o de bajo CI que contribuirían a explicar el fracaso lector. Las diferencias significativas en las puntuaciones del RAVEN en $2^{\circ}$ y $4^{\circ}$ curso entre escolares con DAL y normolectores, son debidas a que en nuestro estudio no excluimos los casos con bajo CI.

En cuanto a las diferencias encontradas de la frecuencia del retraso lector por curso, cabe señalar que el porcentaje es superior en $2^{\circ}(15.2 \%)$ que en los cursos posteriores $\left(9.3 \%\right.$ en $4^{\circ} \mathrm{y}$ $10.9 \%$ en $6^{\circ}$ ), si bien esta diferencia se reduce cuando se considera el nivel en los mecanismos de identificación de palabras. La explicación que encontramos más pertinente considera que algunos alumnos tardan más tiempo en aprender a leer y ello reduce la cantidad de práctica lectora acumulada si se compara con sus compañeros más precoces. La aparición de un mayor porcentaje de casos en los que el fracaso en el test de Eficiencia lectora no se acompaña de un fracaso en la prueba de lectura de pseudopalabras ( $25 \%$ en $2^{\circ}$ frente a $13 \%$ en $4^{\circ}$ y $7 \%$ en $6^{\circ}$ ) apoya claramente esta explicación. Estos aprendices ya dominan el código pero todavía no reconocen las palabras eficientemente. Cabe prever, por tanto, que algunos de los escolares identificados con retraso lector en $2^{\circ}$ curso vayan recuperándose con una mayor experiencia lectora, de forma que el porcentaje de casos con DAL resulte equivalente a lo largo de la escolaridad. Nuestros resultados en $4^{\circ}$ y $6^{\circ}$ muestran que alrededor del $10 \%$ de los escolares mantiene sus dificultades sin lograr alcanzar en muchos casos ni siquiera el nivel lector normal de $2^{\circ}$ curso.

Es importante señalar también la mayor frecuencia de malos lectores en los grupos de repetidores, de TDAH y de escolares con algún problema en el desarrollo del lenguaje, indicativo de que estos factores se agregan como causas de dificultad para aprender a leer.

Al explorar, en la $2^{\text {a }}$ fase de la evaluación, los mecanismos de identificación de las palabras, tratamos de establecer las causas potenciales próximas del retraso observado en la lectura y comprensión de las frases del test de eficiencia lectora, ya que es el conocimiento de dichas causas lo que puede orientar sobre programas de intervención que se dirijan a mejorar el rendimiento de estos niños. La lectura de textos depende del éxito en la identificación de las palabras que los componen, palabras que en la medida que sean reconocidas de forma automática y sin esfuerzo no consumirán los recursos imprescindibles para el trabajo de comprensión. De ahí que las medidas de reconocimiento ortográfico, evaluadas a través de las pruebas de decisión y dictado, permitan detectar dificultades en la adquisición de representaciones ortográficas de las que depende la lectura fluida. Por otro lado, la lectura de pseudopalabras muestra la capacidad para aplicar correspondencias grafofonológicas que será necesaria para identificar cualquier palabra no familiar de la que no se disponga representación ortográfica. Las dificultades en la aplicación de este mecanismo de identificación por vía fonológica compromete seriamente el progreso en el aprendizaje de la lectura, ya que a través de su aplicación repetida sobre las nuevas palabras se van elaborando las representaciones ortográficas que han de permitir la identificación directa y automática de las mismas (véase Share, 1995, para una elaboración detallada de este proceso de auto enseñanza). Este modelo explica que la mayor parte de los lectores retrasados identificados en nuestro estudio, muestren problemas no sólo en el mecanismo fonológico sino también en el ortográfico (Tipo 3 ), siendo muy pocos los casos en los que el léxico ortográfico parece desarrollarse a pesar de las dificultades en el mecanismo fonológico (Tipo 1).

Así pues, los resultados obtenidos en la $2^{\mathrm{a}}$ fase de nuestro trabajo muestran que la gran mayoría de los lectores retrasados presentan un déficit marcado, respecto a los normolectores, que afecta a los dos mecanismos de identificación de palabras. El porcentaje es de $67 \%$ en $2^{\circ}$ pero alcanza $85 \%$ en $4^{\circ}$ y permanece estable en $6^{\circ}$. Este resultado, tal como hemos señalado, 
se debe principalmente a la evolución de la frecuencia de niños que presentan un déficit únicamente a nivel ortográfico que pasa de $18 \%$ a $7 \%$ entre $2^{\circ}$ y $6^{\circ}$ año, y sugiere que la adquisición de representaciones ortográficas, que en última instancia depende de la calidad de los mecanismos fonológicos, presenta diferencias individuales importantes. A fonología idéntica, algunos aprendices parecen necesitar más práctica que otros para almacenar información ortográfica. Estas diferencias individuales explican porqué el déficit (exclusivamente) ortográfico es más aparente en $2^{\circ}$ año y disminuye progresivamente, de forma que la proporción de escolares que muestran problemas en las dos competencias de base tiende a estabilizarse alrededor de $85 \%$.

En resumen, los mecanismos básicos de identificación de palabras escritas, deficientes en los disléxicos son la causa inmediata de sus problemas de lectura de nivel superior (frases, textos). La descodificación deficiente, lenta e imprecisa, no permite elaborar representaciones ortográficas de las palabras porque esta elaboración pasa necesariamente por la descodificación (mecanismo de autoenseñanza, Share, 1995). La ausencia de representaciones ortográficas de las palabras explica las deficiencias de los disléxicos en la tarea de dictado y decisión ortográfica que, recuérdese, examinaba precisamente el léxico ortográfico de los participantes.

Nuestra investigación aborda el problema de la detección y evaluación de escolares con dificultades específicas de aprendizaje de la lectura. Las características de la muestra estudiada: todo el alumnado de los cursos $2^{\circ}, 4^{\circ}$ y $6^{\circ}$ de primaria, escolarizado en centros públicos y concertados de un municipio, nos permite una buena aproximación para valorar la incidencia del problema en la población escolar en general. El valor de prevalencia encontrado de las DAL se ajusta al encontrado en otros estudios de lenguas de ortografía trasparente cuando se usan criterios similares (Ziegler, Perry, Ma-Wyatt, Ladner y SchulteKorne, 2003). Este valor resulta ligeramente más elevado que el encontrado por Jiménez et al. (2009) en un trabajo similar en castellano, pero la inclusión en nuestro estudio de los casos con CI bajo y de todos los casos con grave retraso lector sin excluir los que también los pudieran tener en ortografía, podría explicar en buena medida esta diferencia.

El procedimiento y los instrumentos de detección y evaluación de la Batería DIS-ESP (Carrillo y Alegría, en preparación) utilizados en este estudio son fáciles de aplicar en el entorno escolar por los orientadores psicopedagógicos en colaboración con el profesorado tutor o especialista. Por otra parte, los resultados obtenidos sugieren que puedan ser adoptados como parte de un protocolo de aplicación generalizada en las escuelas. El protocolo DIS-ESP incluye una tercera fase de evaluación que explora el procesamiento fonológico de base a diferentes niveles. Tal es el caso de las habilidades necesarias para la percepción, mantenimiento y recuperación de representaciones fonológicas (véase, Carrillo, 2010) y que pueden ser evaluadas a través de tareas de discriminación fonética, memoria fonológica, nombrado rápido o fluidez verbal, entre otras. También, y de forma más directamente relacionada con el mecanismo fonológico de lectura, la evaluación de la conciencia fonológica, que permite segmentar en sílabas y fonemas las palabras, constituye el indicador más potente del riesgo de fracasar en la adquisición de dicho mecanismo básico de lectura. Datos, no presentados en este trabajo, en este tipo de pruebas y que forman parte de la $3^{\mathrm{a}}$ fase de nuestro estudio, ayudan a precisar el origen cognitivo de las dificultades y qué escolares están en mayor riesgo de persistir en sus dificultades de aprendizaje (véase, Carrillo y Alegría 2009b).

\section{Referencias}

1. Alegría, J. (2006). Por un enfoque psicolingüístico del aprendizaje de la lectura y sus dificultades -20 años después-. Infancia y Aprendizaje, 29, 93-111.

2. Calvo, A. R. (1999). Adquisición de la lectura en lengua castellana: perfiles cognitivos de aprendices con dificultades. Tesis doctoral. Departamento de Psicología Básica y Metodología. Universidad de Murcia, España.

3. Carrillo, M.S. (2010). La evaluación de la dislexia evolutiva. En M. Carrillo y A.B. Domínguez (Coord.), Dislexia y sordera: Líneas actuales en el estudio de la lengua escrita y sus dificultades (pp. 111-132). Málaga: Aljibe.

4. Carrillo, M.S. y Alegría, J. (2009a). Mecanismos de identificación de palabras en niños disléxicos en español: ¿Existen subtipos? Ciencias Psicológicas, 3, 135-152.

5. Carrillo, M.S. y Alegría, J. (2009b). Exploración de habilidades fonológicas en escolares disléxicos: teoría y práctica. Revista de Logopedia, Foniatría y Audiología, 29, 105-120.

6. Carrillo, M.S y Marín, J. (1997). Test de Eficiencia Lectora - TECLE. Publicado en A. Cuadro, D. Costa, D. Trias y P. Ponce de León, (2009) Evaluación del nivel lector. Manual técnico del test de Eficacia Lectora (TECLE). Uruguay: Prensa Médica Latinoamericana.

7. Cuetos, F., Rodríguez, B. y Ruano, E. (1996). PROLEC: Evaluación de los procesos lectores. Madrid: TEA Ediciones.

8. Cuetos, F., Suárez-Coalla, P. y Davies, R. (2010). La adquisición de la lectura en castellano. En M. Carrillo y A.B. Domínguez (Coord.), Dislexia y sordera: Líneas actuales en el estudio de la lengua escrita y sus dificultades (pp. 6173). Málaga: Aljibe.

9. Davies, R., Cuetos, F. y González-Seijas, R. (2007). Reading development and dyslexia in a transparent orthography: a survey of Spanish children. Annals of Dyslexia, 57, 179198. http://dx.doi.org/10.1007/s11881-007-0010-1

10. Flynn, J.M. y Rahbar, M.H. (1994). Prevalence of reading failure in boys compared with girls. Psychology in the Schools, 31, 66-7. http://dx.doi.org/10.1002/1520-6807 (199401)31:1<66::AID-PITS2310310109>3.0.CO;2-J

11. Jiménez, J. y Hernández, E. (2000). Word identification and reading disorders in the Spanish language. Journal of 
Learning Disabilities, 33, 44-60.

12. Jiménez, J., Guzmán, R., Rodríguez, C. y Artiles, C. (2009). Prevalencia de las dificultades específicas de aprendizaje: la dislexia en español, Anales de psicología, 25, 78-85.

13. Jiménez, J. y Ramírez, G., (2002) Identifying subtypes of reading disability in the Spanish language. Spanish Journal of Psychology, 5, 3-19.

14. LOE (2006). Ley Orgánica 2/2006, de 3 de mayo, de Educación (BOE 4-5-2006).

15. Martínez, J. (1995). La dislexia evolutiva: un enfoque neuropsicológico. Tesis doctoral. Universidad de Salamanca.

16. Martínez, J. y García, E. (2004): Diccionario de frecuencias del castellano escrito en niños de 6 a 12 años. Salamanca: Publicaciones Universidad Pontificia.

17. Perfetti, C. (1985). Reading ability. New York: Oxford University Press.

18. Serrano, F. (2005). Disléxicos en español: Papel de la fonología y la ortografía. Tesis doctoral. Universidad de Granada.

19. Serrano, F. y Defior, S. (2005). Dyslexia in Spanish: the state of the matter. Electronic Journal of Research in Educational Psychology, 2, 13-34.

20. Share, D.L. (1995). Phonological recoding and self-teaching: sine qua non of reading acquisition. Cognition, 55, 151-218.
21. Share, D.L. y Stanovich, K.E. (1995). Cognitive processes in early reading development: Accommodating individual differences into a model of acquisition. Issues in Education, 1, 1-57. http://dx.doi.org/10.1016/0010-0277(94)00645-2

22. Snowling, M. (2001). From language to reading and dyslexia. Dyslexia, 7, 37-46. http://dx.doi.org/10.1002/dys.185

23. The International Dyslexia Association (IDA) (2002). What is dyslexia? (Adopted by the IDA Board of Directors, Nov. 12, 2002) [Online]. Disponible en: http://www.dyslexia-ca. org/dyslexiadefinition.html (consultado en abril 2011).

24. Ziegler JC, Perry C, Ma-Wyatt A, Ladner D y SchulteKorne G. (2003). Developmental dyslexia in different languages: language-specific or universal? Journal of Experimental Child Psychology. 86, 169-93. http://dx.doi. org/10.1016/S0022-0965(03)00139-5

Fecha de recepción: 18 de marzo de 2011 Fecha de recepción de la versión modificada: 2 de junio de 2011 Fecha de aceptación: 14 de junio de 2011 